\title{
Initial Data-Element Selection for the Evaluation of Picture Archiving and Communication System Performance
}

\author{
Hiroshi Kondoh, Junpei Ikezoe, Kiyonari Inamura, Chikazumi Kuroda, and Takahiro Kozuka
}

\begin{abstract}
The completely new, hospital-wide picture archiving and communication system (PACS) now being implemented at Osaka University Hospital is described elsewhere in this issue. ${ }^{\top}$ This paper lists the many studies of the department and hospital that were performed before the PACS for the purpose of identifying data elements for use in evaluating a PACS system. A second purpose of the initial data-element collection was to assist in the overall Osaka University PACS design. Selected studies from this work are presented here.
\end{abstract}

Copyright 1994 by W.B. Saunders Company

KEW WORDS: picture archiving and communication system (PACS), technology assessment, effectiveness.

$\mathbf{T}$ HE PRE-PACS MANUAL system was quantitatively measured. Definitions of variables, parameters, formulas, and methods of measurement were selected to be the same, or nearly so, for comparison of the same measurements obtained before and after the use of the PACS. ${ }^{1}$ These definitions were carefully selected with the purpose of adapting this methodology of PACS technology assessment at other hospitals. The final target of the technology assessment is the measurement of clinical effectiveness, rather than the measurement of hardware and software in terms of speed, capacity, and the resolution of images. ${ }^{2,3}$ Such a method of PACS evaluation appears to be the most easily understandable approach to establishing the effectiveness of PACS. On the other hand, to optimize the system design of PACS, careful selection of data elements and methods of measurement are also necessary.

Table 1 lists the initial data elements and the studies that were performed over a 2-year period. Selection of these elements for evaluation of PACS performance and/or optimization of PACS design is also tabulated. The results of some of these studies are presented in this paper as marked in Table 1.

\section{DISTRIBUTION OF IMAGING-STUDY USAGE}

A detailed study of the image usage on each ward and in each clinic in the hospital was undertaken (Table 1). The number of each type of radiologic study ordered for the patients from each location was tabulated according to modality and the number of radiographs. In addition, the division of routine versus urgent studies was also recorded. Ratios of urgent to nonurgent cases for each exam type were determined.

A number of these studies loaned to each care location were also tabulated by modality and by the number of radiographs loaned for each exam.

The number of view boxes $(14-\times 17$-in display panels) were counted in every clinic and ward in the hospital. These numbers are shown in the top half of Fig 1. The bottom half of Fig 1 shows the number of imaging studies ordered for various locations expressed as the number of radiographs taken.

An important ratio was defined from the data of Fig 1 to measure the volume and intensity of light-box usage in each area. The number of radiographs taken in an area divided by the number of view boxes present was the intensity measure. These ratios validate the workload and utility of the view boxes in the various locations. The comparison shows only a loose correlation between volume and number of view boxes. Departments with a large number of radiographs on hand did not necessarily have a large number of view boxes and vice versa.

This type of data was used in the system design for the PACS to determine the correct distribution and to estimate the correct performance of display workstations. Given the cost of these workstations, the data was useful to allow planning for a minimal number of workstations to be deployed where they would be most needed. Matching the distribution to usage of workstations is important because the high cost

From the Department of Radiology, School of Medicine; and the Department of Radiological Technology and Biomedical Engineering, School of the Allied Health Sciences, Faculty of Medicine, Osaka University, Osaka, Japan.

Address reprint requests to Hiroshi Kondoh, MD, Department of Radiology, Osaka University Medical School, 2-2, Yamada-Oka, Suita, Osaka 565, Japan.

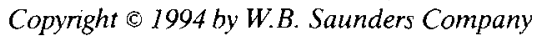

0897-1889/94/0704-0009\$3.0010 
Table 1. Initial Data-Element Selection and its Contribution to Evaluating PACS and/or PACS Design

\begin{tabular}{|c|c|c|c|c|}
\hline Classification & Data Item & $\begin{array}{l}\text { Contribution } \\
\text { to Evaluation }\end{array}$ & $\begin{array}{l}\text { Contribution } \\
\text { to Design }\end{array}$ & $\begin{array}{l}\text { Figure } \\
\text { Number }\end{array}$ \\
\hline \multirow[t]{6}{*}{ Distribution of imaging study usage } & Number of radiologic orders & * & * & \\
\hline & Number of radiographs taken & * & * & 1 \\
\hline & Division of routine versus urgent & * & * & \\
\hline & Number of image loan & * & * & \\
\hline & Number of view boxes & * & * & 1 \\
\hline & Number of outside radiographs & * & * & 2,3 \\
\hline Flow study & $\begin{array}{l}\text { Flow studies on patients, images, orders, requisi- } \\
\text { tions, reports, etc. }\end{array}$ & * & * & 4 \\
\hline \multirow[t]{7}{*}{ Time study } & Time required for order collection & * & * & \\
\hline & Time required for image delivery & * & $*$ & \\
\hline & Time for imaging process & * & * & 5 \\
\hline & Time until entry of film count and interpretation & * & * & 6 \\
\hline & $\begin{array}{l}\text { Time around time from order entry until report/ra- } \\
\text { diographs return }\end{array}$ & * & & 7 \\
\hline & Distribution of number of imaging studies in a day & * & * & 8,10 \\
\hline & Distribution of number of cases interpreted in a day & * & & 9,10 \\
\hline \multirow[t]{3}{*}{ Reporting analysis } & Number of letters in a report & * & * & 11 \\
\hline & Time spent in generating report & * & & \\
\hline & Time for writing letters in reporting & * & & \\
\hline \multirow[t]{2}{*}{ Cost analysis } & $\begin{array}{l}\text { Change in the composition of cost of image diag- } \\
\text { nosis }\end{array}$ & * & & 12 \\
\hline & $\begin{array}{l}\text { Change in cost for films, contrast medias, and } \\
\text { radiopharmaceuticals }\end{array}$ & * & & \\
\hline
\end{tabular}

* Denotes a contribution to evaluation or design of PACS.
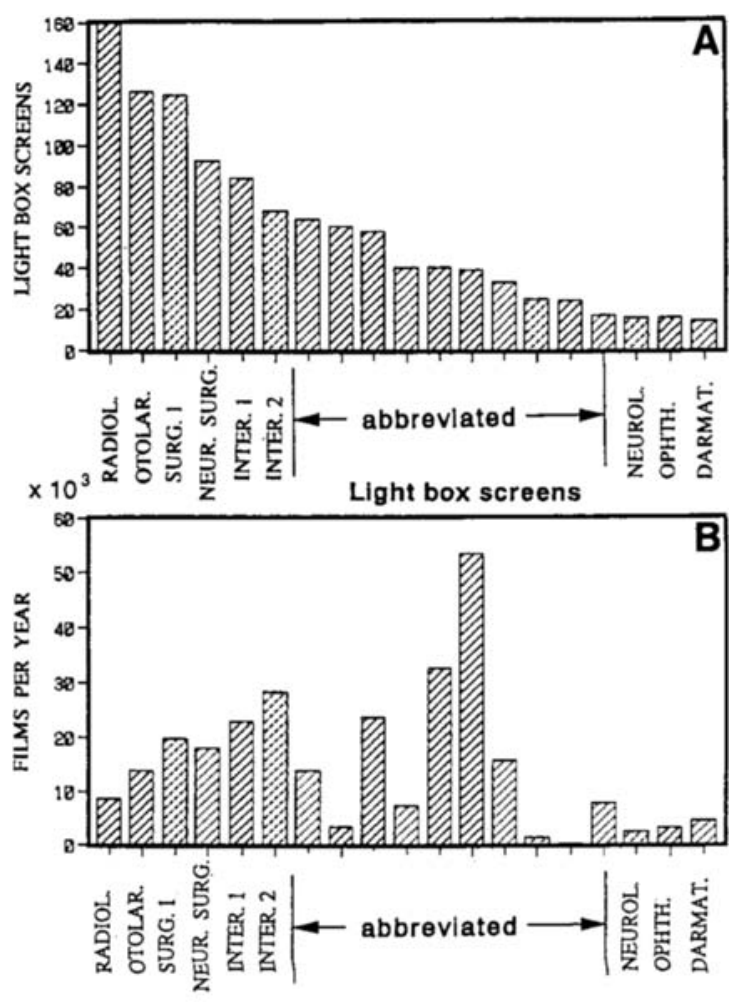

radiographs per year

Fig 1. Light-box screens in each department $(A)$ and the number of radiographs taken per year by each department (B). of these stations determines that the number available for display would be substantially less than the number of view boxes now present.

\section{OUTSIDE RADIOGRAPHS}

Osaka University Hospital is an important tertiary-care center that receives referrals from many other hospitals. In addition, a substantial portion of the hospital beds are for longer-term care. These two factors result in a large number of outside radiographs coming with patients to the hospital.

The number of such outside radiographs for inpatients and outpatients was counted over a 5-day period. The results for sample care areas are shown in Fig 2, broken down into those stored for continuing use and those returned to the originating-care facility. The distribution of the volume of such outside radiographs and the volume of Osaka University radiographs was determined and is shown in Fig 3A. The distribution and the fluctuations of the number of outside radiographs received by modality and day of week during the 5-day-workweek study period, as well as for the total of all 5 days, is shown in the graph in Fig 3B. This data was also directly useful in planning the PACS configura- 
Fig 2. Number of outside radiographs brought to each department in a day as the average of those in 5 days (Monday, February 3 to Friday. February 7. 1992). $\square$, Stored film; 6 , returned film.

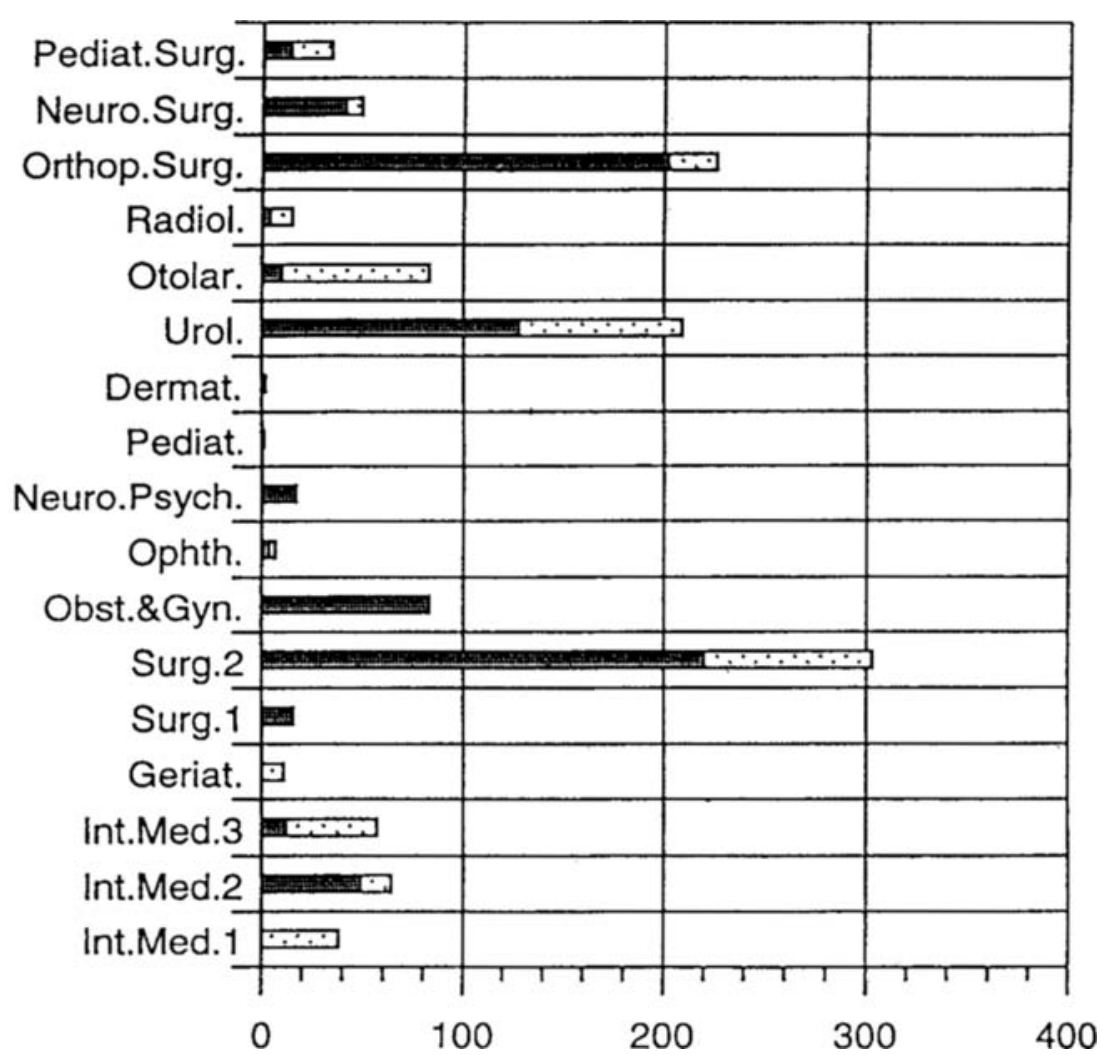

image delivery, time required for imaging process (Fig 5), time until entry of film count and interpretation (Fig 6), the turn-around time from a request for a study until the report was received in the care area (Fig 7), time spent by the radiologist in creating or generating the radiology report, time for writing letters in a reporting.

It is clear that most of the imaging process is in time within 5 minutes and $70 \%$ of them are in 10 minutes, even in pre-PACS environment. Also, Fig 6 tells that $76 \%$ of the radiographs are entered into the system within 8 hours after the imaging process is over with their interpretation and the film count. However, the turn-around time distributes to 39 hours with the peak at 29 hours as shown in Fig 7. Even if we take the case of 16 hours in Fig 7, it is much longer than 10 minutes plus 8 hours as shown in Figs 5 and 6 . This evidence clearly tells that the time required for order collecting and the time required for image delivery are very long, extending from 8 hours to 31 hours. We measured them, and the data exactly verified above figures. These figures suggest that only joint opera- 


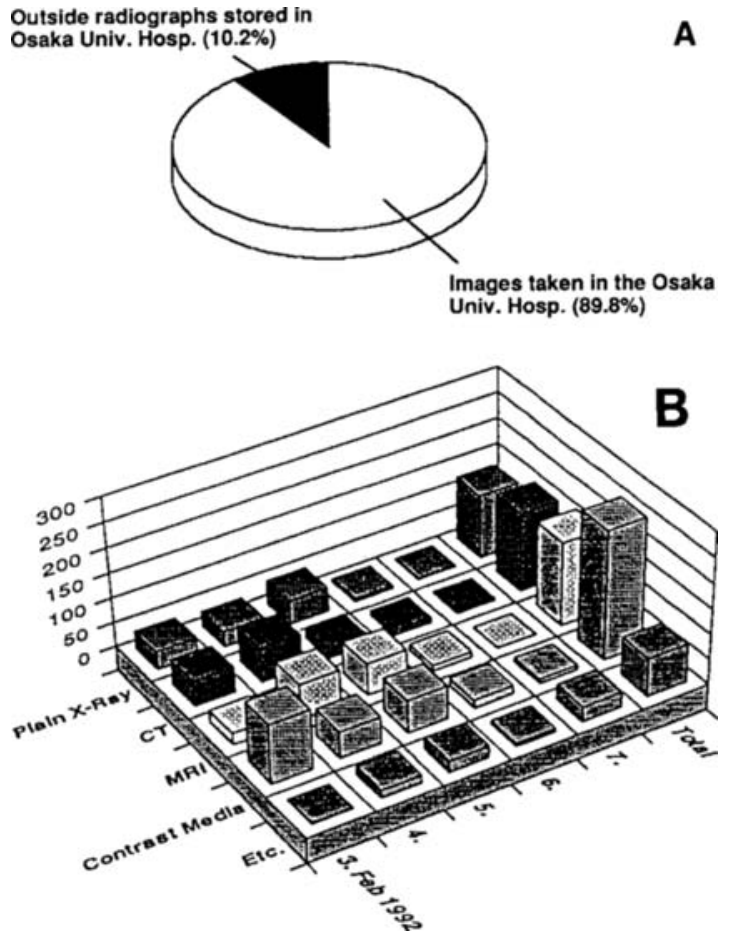

Fig 3. (A) The distribution of the volume of outside radiographs and the volume of inside radiographs taken at Osaka University Hospital in 5 days (Monday, February 3 to Friday, February 7, 1992). (B) The number of outside radiographs stored in the hospital in terms of modalities and days during the week.

tion of PACS with HIS and RIS will be effective in shortening the turn-around time.

The distribution of imaging studies done (counted as the number of radiographs) during the working day was tabulated. The results are shown in Fig 8. Several different peak periods at various hours of the day are clearly identifiable.

The number of cases interpreted by hour of day were also obtained. Figure 9 shows this data expressed as the number of radiographs read for both new studies and comparison studies. Figure 10 shows these data superimposed with the curve of when the imaging studies were done. An $\approx 30$-minute delay in the interpretation of cases was identified in the morning. In the afternoon, the delay became $\sim 60$ minutes. The maximum volume of interpretations occurred between 3 and 4 PM. The identification of peaks and the quantification of the number of studies handled were directly useful in planning for peak network traffic and system work loads.

In both ordering and reporting for radiologic examinations, Japanese physicians use a combination of text and schematics. The number of words and characters and schematics was counted in randomly extracted samples of radiologic requisitions and reports. Figure 11 shows the number of the letters counted in a report. This data was taken for the purpose of observing the data even in a post-PACS environment and of maintaining the number of words and quality of reports.

\section{COST ANALYSIS}

The total cost of operation of Osaka University Hospital from the period 1982 to 1991 was compared with certain supply costs for radiologic imaging during the same time period. These costs included radio pharmaceuticals, films, and contrast media. The ratio of the radiology supply cost to the total was calculated and is shown for inpatients and outpatients and all patients in Fig 12. Although the decreasing trend in radiology costs is real, it has been affected by such things as revisions of the cost tables used by various health-insurance systems. Despite this, it is anticipated that a positive impact of PACS will further decrease the cost of radiologic supplies in the future.

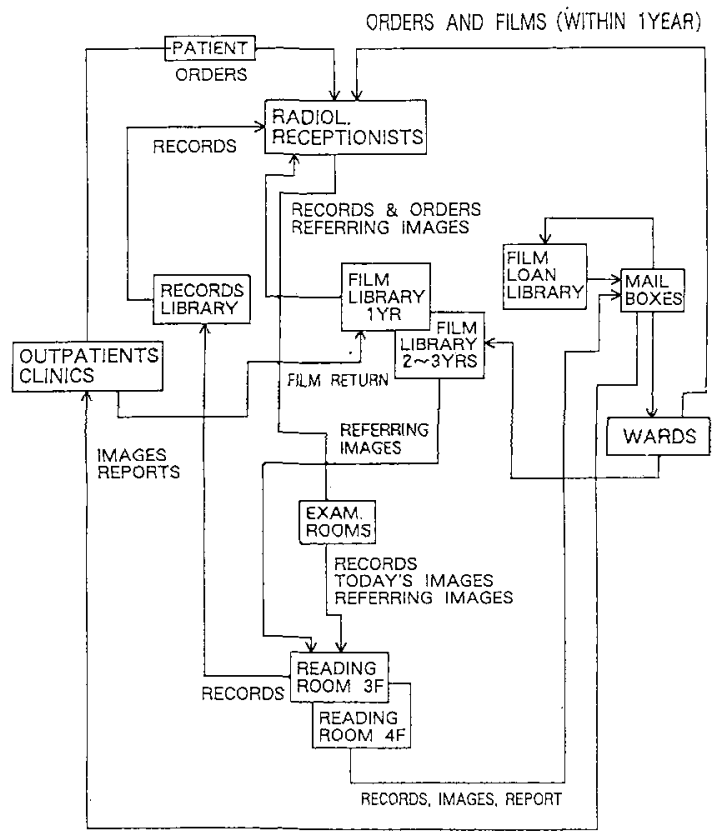

Fig 4. Outline of the result of flow study of images, orders, and reports in Osaka University Hospital. 


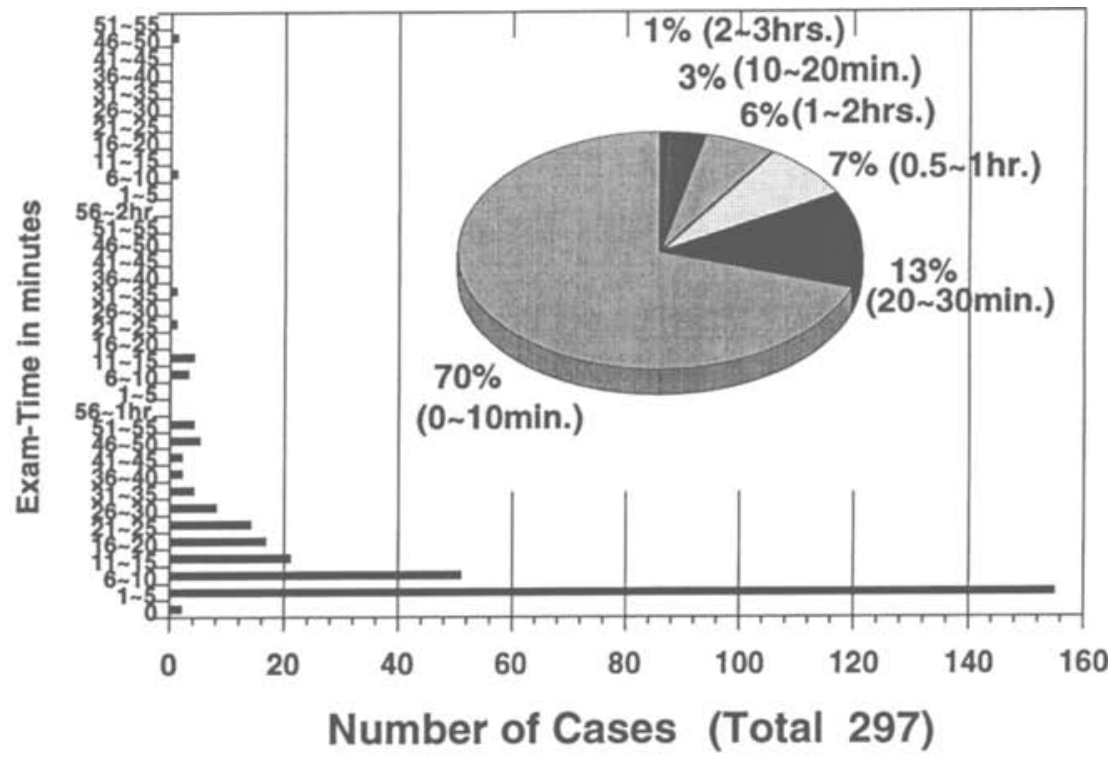

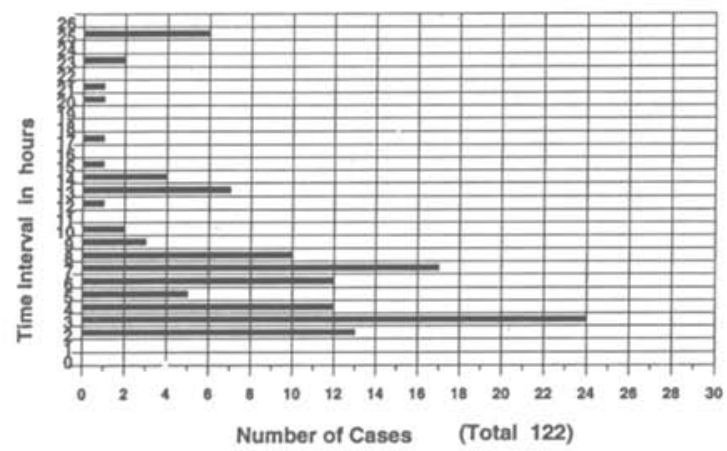

Fig 6. Time until entry of film count and interpretation.

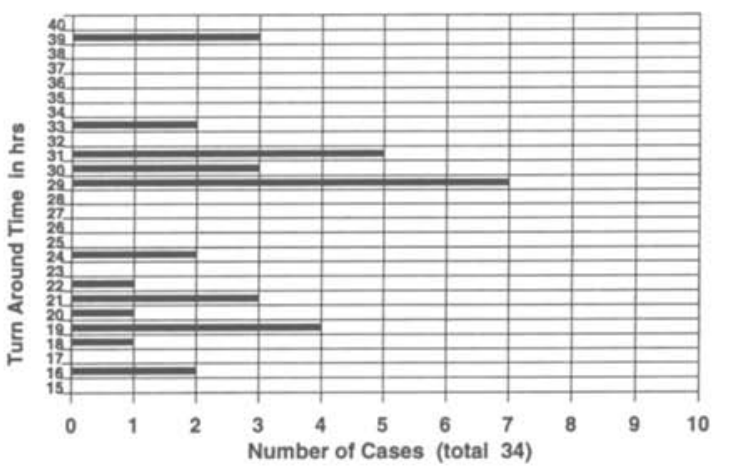

Fig 7. Turn-around time at wards.

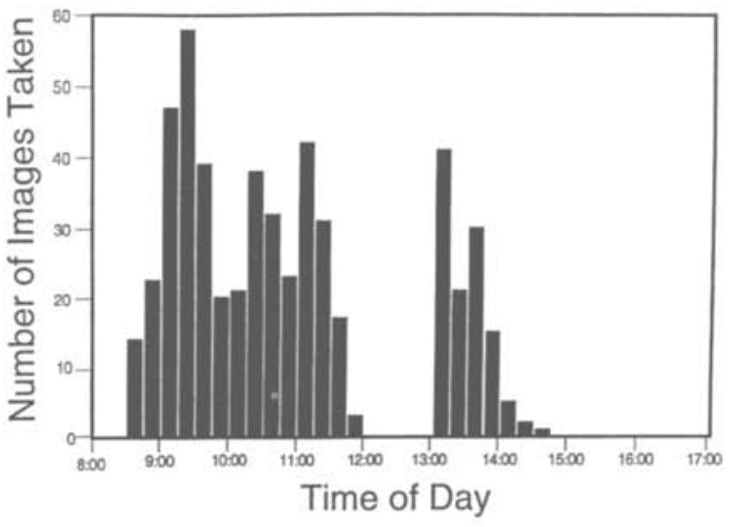

Fig 8. Number of images taken in terms of time of day as the average over 5 days.

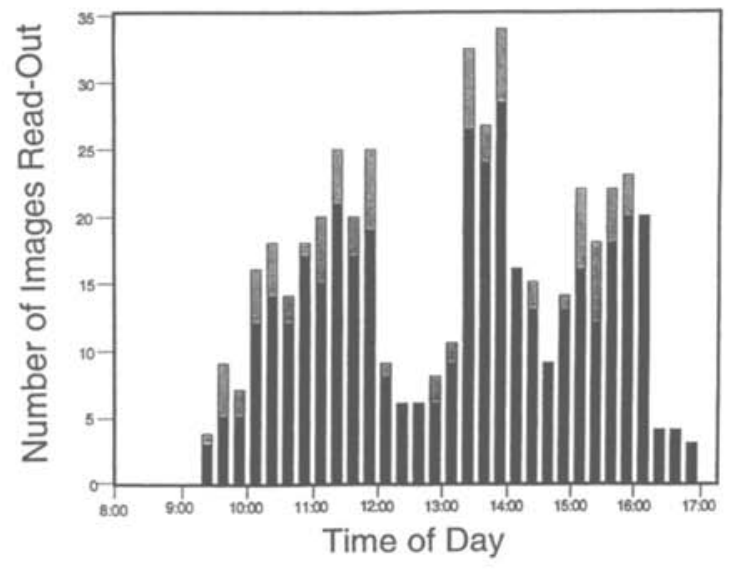

Fig 9. Number of images read out in terms of time of day as the average over 5 days. (घ), read-out images that were taken on that day; ( $\square$ ), referred images that were taken on previous days. 


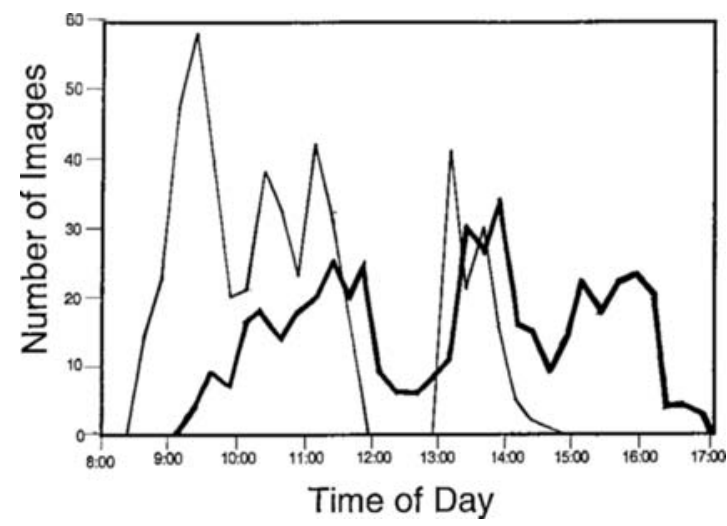

Fig 10. Comparison of number of images read out (-) with number of images taken (-) in terms of time of day. The delays of peaks between two curves are shown.

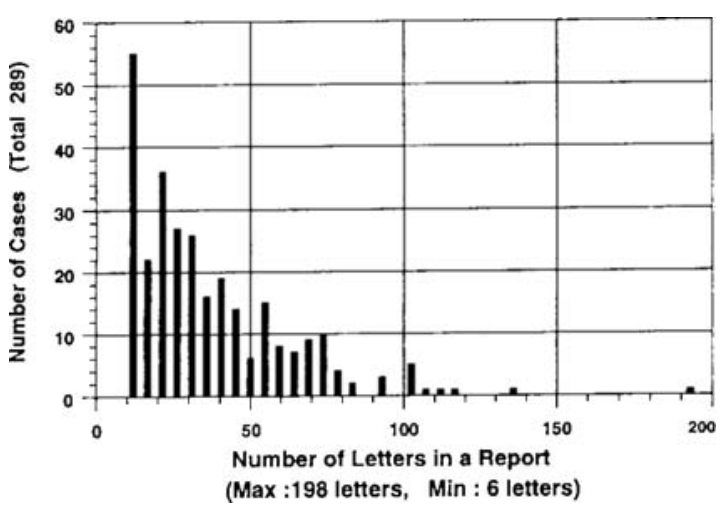

Fig 11. Number of letters in a report.

\section{DISCUSSION}

Table 1 sums up the studies that were performed in our pre-PACS environment. Aster-

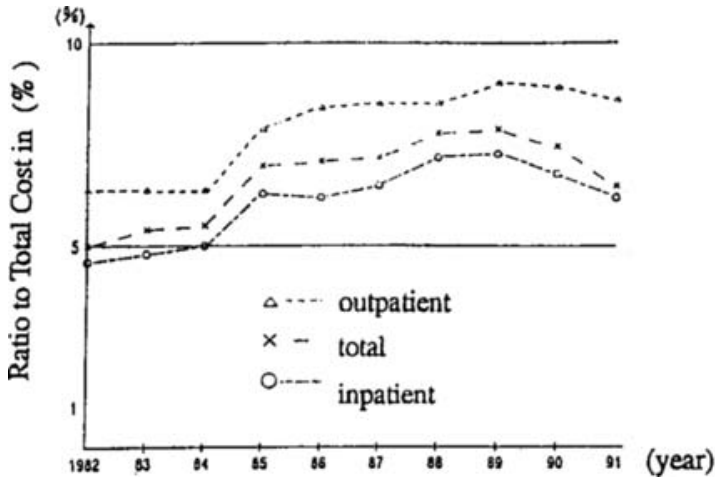

Fig 12. Change in the composition of cost of image diagnosis in Osaka University Hospital from 1982 to 1991.

isks in the column "Contribution to Evaluation" show that these items must be again measured in our post-PACS environment and can be adopted in other hospitals to evaluate PACS by pre-post-PACS measurement. Asterisks in the column "Contribution to Design" indicate that these studies contributed to our PACS design and will contribute to PACS in other hospitals.

\section{REFERENCES}

1. Kondoh H, lkezoe J, Inamura $\mathbf{K}$, et al: Developing a new PACS for the new Osaka University Hospital. I Digit Imaging 4:172-176, 1994

2. Inamura $\mathrm{K}$ : System design and technology assessment: Proceedings of IMAC '91. Los Alamos, CA, IEEE Computer Society, 1991, 154-166

3. Kondoh $\mathrm{H}$, Ikezoe J, Inamura $\mathrm{K}$, et al: A comparison of conventional screen-film radiography and hard copy computed radiography in detection of interstitial lung disease. J Digit Imaging 4:193-195, 1994 